\title{
Miocardiopatía de Tako tsubo y movimiento sistólico anterior de válvula mitral
}

\author{
Tako tsubo myocardiopaty and sistolic anterior movement \\ of mitral valve
}

Emilia Schlackํㅗ Fernando Aranda²

\begin{abstract}
Tako tsubo cardiomyopathy associated with left ventricular outflow tract obstruction (LVOT) is a rare cause of persistent perioperative hypotension. One of the causes of this association is the systolic anterior motion (SAM) of the mitral valve. We report a case of a 67-year-old woman who, after undergoing liver segmentectomy because of metastasis, presents post-operative hypotension that is difficult to manage. Upon evaluation with a transthoracic echocardiogram, the diagnostic suspicion of Tako tsubo syndrome associated with LVOT obstruction secondary to SAM was raised. As initial therapy, a volume expander, in association with propanolol and phenylephrine were administred, achieving partial hemodynamic response. Later, she evolved with signs of heart failure and was transferred to the intensive care unit for management. In this unit, non-invasive ventilatory support, diuretic and vasopressor therapy were required, achieving favorable results on the second post-operative day. Echocardiography was essential to make the differential diagnosis against a persistent post-operative hypotension.
\end{abstract}

\section{RESUMEN}

La cardiomiopatía de Tako tsubo asociada a obstrucción del tracto de salida del ventrículo izquierdo (TSVI) es una causa poco frecuente de hipotensión persistente en el perioperatorio. Uno de los motivos de esta asociación es el movimiento anormal sistólico (SAM) de la válvula mitral. A continuación, presentamos el caso de una mujer de 67 años que, tras ser sometida a segmentectomía hepática por metástasis, presenta cuadro de hipotensión postoperatoria

\section{Key words:}

Tako tsubo

myocardiopathy, sistolic anterior motion mitral valve

\section{Palabras clave:}

Cardiomiopatía de Tako tsubo,

Movimiento sistólico anterior de válvula mitral

\footnotetext{
Médico residente de Anestesiología, Universidad de Valparaíso. Valparaíso, Chile.

2 Médico Anestesiólogo Cardiovascular, Jefe de Departamento de Anestesiología, Universidad de Valparaíso. Valparaíso, Chile.
}

Fecha de recepción: 18 de marzo de 2020

Fecha de aceptación: 04 de abril de 2020

\section{ORCID}

https://orcid.org/0000-0002-7750-689X

\section{Correspondencia:}

Emilia Schlack

Email: emiliasb@gmail.com 
de difícil manejo. Al ser evaluada con ecocardiograma transtorácico se plantea la sospecha diagnóstica de síndrome de Tako tsubo asociado a obstrucción del TSVI secundario a SAM. Como terapia inicial se realizó aporte de volumen, en asociación con propanolol y fenilefrina, logrando parcial respuesta hemodinámica. Posteriormente, evolucionó con signos de insuficiencia cardiaca y fue trasladada a unidad de cuidados intensivos para su manejo. En esta unidad requirió apoyo ventilatorio no invasivo, terapia diurética y vasopresora, logrando resultados favorables al segundo día postoperatorio. La ecocardiografía fue fundamental para realizar el diagnóstico diferencial frente a un cuadro de hipotensión postoperatoria persistente.

\section{Introducción}

a cardiomiopatía de Tako tsubo, también conocida como cardiomiopatía de estrés, es un cuadro transitorio de disfunción ventricular que ha sido asociada a altos niveles de catecolaminas circulantes, ya sea secundario a estados de estrés intenso físico y/o emocional, o bien, al aporte exógeno de catecolaminas[1]. Se caracteriza por presentarse en ausencia de lesiones coronarias que expliquen el cuadro[2],[3].

Movimiento anormal sistólico o "Systolic anterior motion" (SAM) de la válvula mitral, corresponde al desplazamiento anterior de uno o ambos velos mitrales, produciendo obstrucción dinámica del tracto de salida del ventrículo izquierdo[4].

Ambos cuadros clínicos presentan factores de riesgo en común, han sido descritos en asociación y como diagnósticos diferenciales a considerar en pacientes con inestabilidad hemodinámica persistente perioperatoria[5]. A continuación, se presenta un caso clínico de hipotensión sostenida intra y post operatoria, secundaria a SAM en el contexto de cardiomiopatía de Tako tsubo.

\section{Caso clínico}

Mujer de 67 años, con antecedentes de hipertensión arterial, cáncer de colon y obesidad (IMC 32). Estudio preoperatorio con ecocardiograma que describe "hipertrofia ventricular izquierda (HVI) moderada con función sistólica global y segmentaria conservadas. Fracción de eyección $72 \%$. Disfunción diastólica tipo 1 1". Electrocardiograma sin alteraciones. Capacidad funcional I.

Ingresa a pabellón de forma electiva para segmentectomía hepática por metástasis secundaria a cáncer de colon. Se inicia cirugía bajo anestesia general y analgesia peridural. Inicialmente recibe dos dosis de bupivacaína 0,3\%, 7 y $8 \mathrm{~mL}$ respectivamente, por catéter peridural y a las 2 horas de cirugía se inicia infusión de levobupivacaína 0,125\% $5 \mathrm{~mL} / \mathrm{h}$. Transcurridas 6 horas desde el inicio de la anestesia se realiza control de laboratorio, destacando hematocrito $25 \%$ y hemoglobina $8,5 \mathrm{~g} / \mathrm{dL}$. Dado lo anterior y, en contexto de hipotensión intraoperatoria, se realiza carga de volumen con $500 \mathrm{~mL}$ de HES $6 \%$ y transfusión de dos unidades de glóbulos rojos concentrados (GRC) más dos unidades de plasma fresco congelado (PFC). Dos horas más tarde finaliza cirugía, paciente es extubada y se suspende infusión de levobupivacaína debido a hipotensión sostenida. Si bien no recibió drogas vasoactivas en infusión durante el periodo intraoperatorio, fueron administrados reiterados bolos de $100 \mathrm{mcg}$ de fenilefrina y efedrina 12 mg (5 y 4 bolos respectivamente). Tras permanecer 8 horas en quirófano Ingresa a Unidad de Recuperación Postanestésica quejumbrosa, taquicárdica (126 lpm) e hipotensa $(86 / 69 \mathrm{mmHg})$, ventilando de forma espontánea sin apremio. Para el manejo del dolor recibe por vía endovenosa morfina $4 \mathrm{mg}$, paracetamol $1 \mathrm{~g}$, ketorolaco $30 \mathrm{mg}$, infusión continua de ketorolaco 90 mg más tramadol 200 mg, además droperidol 1,25 $\mathrm{mg}$ y ondansentron $4 \mathrm{mg}$ para manejo de náuseas y vómitos. A pesar de tratamiento analgésico paciente se mantiene taquicárdica, hipotensa, refiriendo dolor epigástrico. Se realiza electrocardiograma de control que evidencia signos de isquemia anterolateral, taquicardia sinusal y conducción auriculoventricular con preexitación ventricular primero permanente y luego intermitente (Figura 1), que genera un QS apical y lateral.

Ecocardiograma transtorácico evidencia hipokinesia anterior severa, hipokinesia difusa medioapical e hiperkinesia basal, sugerentes de síndrome de Tako tsubo (Figura 2). Además, se observó un SAM y obstrucción del TSVI con gradiente de hasta $154 \mathrm{mmHg}$ (Figura 3). El control enzimático muestra elevación de 


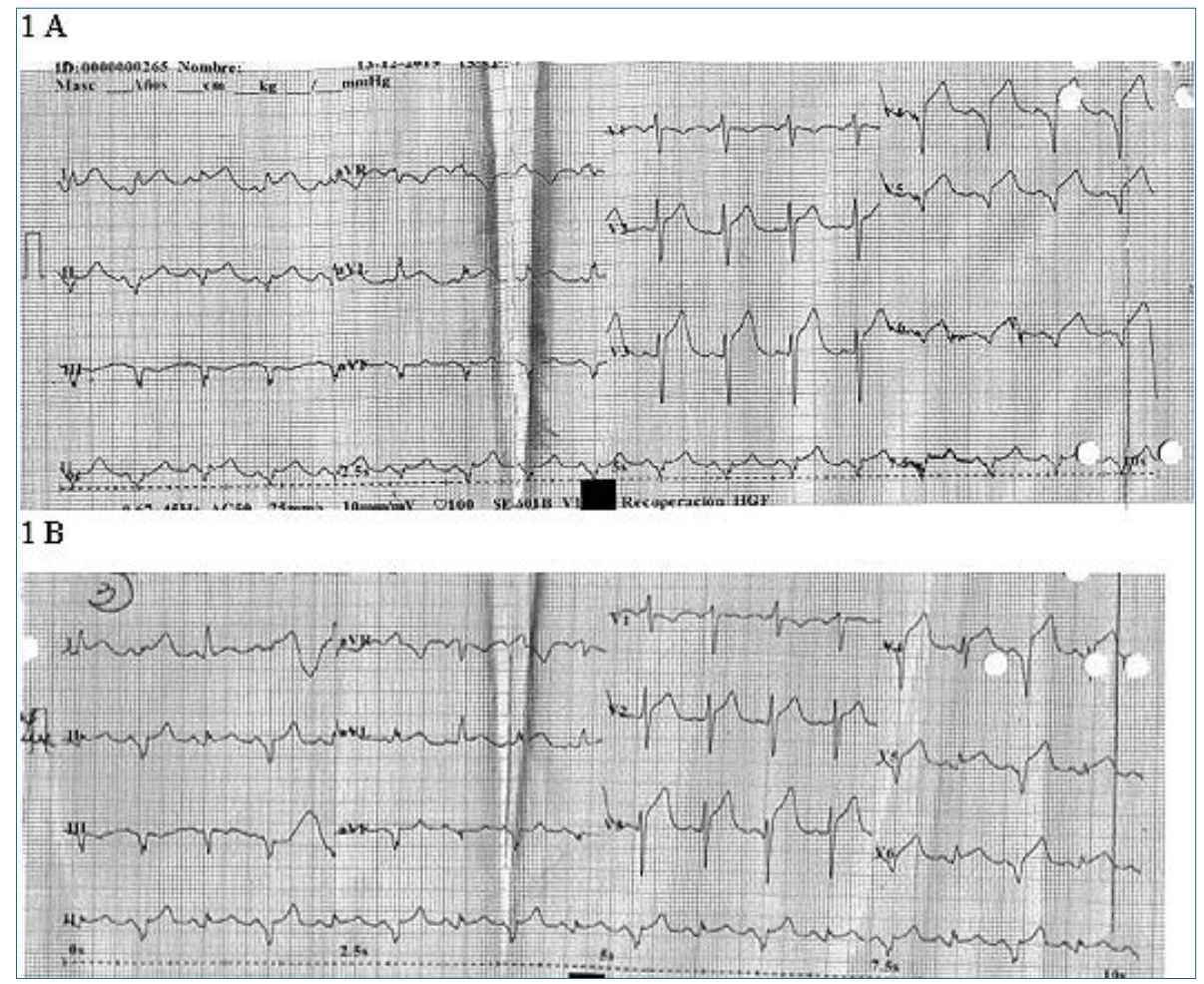

Figura 1. Electrocardiograma (EKG). 1A. EKG con conducción auriculoventricular con preexitación ventricular permanente. 1B. EKG con conducción auriculoventricular con preexitación ventricular intermitente. Obtenidos con 5 minutos de diferencia.

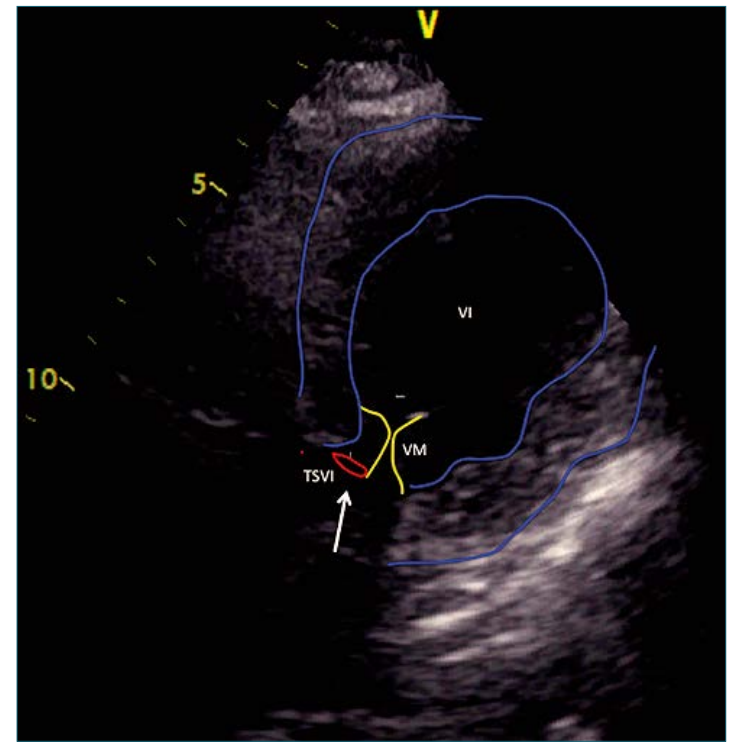

Figura 2. Obstrucción del tracto de salida del ventrículo izquierdo (en rojo, TSVI) (flecha blanca) secundario a SAM de la válvula mitral (en amarillo, VM). Además, imagen de balonamiento del VI típica de la cardiomiopatía de Tako tsubo (borde azul, interno: endocardio; externo: epicardio).

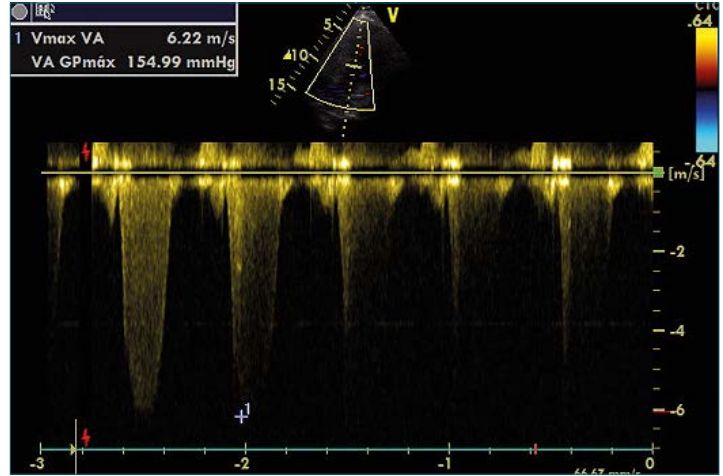

Figura 3. Ecocardiografía transtorácica, ventana apical 4 cámaras: a nivel de la válvula aórtica se cuantifica flujo transvalvular de $6,22 \mathrm{~m} / \mathrm{s}$, calculando un gradiente máximo de $155 \mathrm{mmHg}$.

troponina ultrasensible 5 veces su valor normal y CK - MB 2 veces su valor normal (valores normales: Troponina US 0,047; CK - MB: 5,0).

Frente a estos hallazgos se realiza nueva transfusión de GRC y PFC, se volemiza con $1.000 \mathrm{~mL}$ más de HES $6 \%$, administrando 2 bolos de fenilefrina de 
100 mcg y 2 bolos de propanolol 0,2 mg. A las tres horas de finalizada la cirugía paciente es trasladada a unidad de cuidados intensivos (UCI), en mejores condiciones generales. Sin embargo, en esta unidad evoluciona con signos de congestión pulmonar, con requerimientos de ventilación mecánica no invasiva, infusión continua de furosemida y noradrenalina durante 48 horas. Posteriormente, evoluciona de forma favorable, sin necesidad de apoyo ventilatorio y requerimientos de oxigenoterapia en disminución. Se trasladó a una unidad de menor complejidad al sexto día postoperatorio.

Tres días después se realiza control ecocardiográfico que se describe como "akinesia anteroseptal distal y apical. HVI con función sistólica levemente deprimida. Fracción de eyección 47\%. Disfunción diastólica tipo 1". A las dos semanas el estudio angiográfico describe "Coronarias sin lesiones y fracción de eyección del ventrículo izquierdo normal".

Tras 22 días desde la intervención paciente es dada de alta con tratamiento antihipertensivo, incluyendo betabloqueo y uso de antiplaquetarios.

\section{Discusión}

La hipotensión postoperatoria es una complicación muy común en pacientes sometidos a cirugía bajo anestesia general y/o regional, siendo las etiologías habituales venodilatación e hipovolemia, secundaria a causas como el efecto de drogas anestésicas, sangrado, inflamación sistémica, anafilaxia, arritmias, falla cardiaca, etc.

Dentro de las causas poco frecuentes en cirugía no cardiaca, se describe la obstrucción dinámica del TSVI secundaria a SAM[6]. Este es un proceso que puede estar determinado por condiciones anatómicas del paciente, por situaciones patológicas, uso de ciertos fármacos y alteraciones de la precarga, poscarga y contractibilidad. Dentro de estos factores, se encuentran: hipertrofia del ventrículo izquierdo, cardiopatía isquémica (asociado a síndrome coronario agudo o síndrome de Takotsubo), disminución de la precarga, disminución de la postcarga y estimulación aguda betaadrenérgica[4].

La cardiomiopatía de Takotsubo, también conocida como miocardiopatía de estrés, se caracteriza por tres aspectos distintivos: 1) disfunción aguda de alguna pared del ventrículo izquierdo (VI); 2) ausencia de obstrucción coronaria que explique el cuadro y 3) recuperación de la función sistólica del VI en días o semanas[2]. Clásicamente se desarrolla en mujeres postmenopáusicas, de edad promedio 68 años, caucásicas[7] y es desencadenado por situaciones de estrés emocional o físico muy intensos. Su evolución clínica habitual es benigna y reversible, sin estar exenta de morbimortalidad. Dentro de las complicaciones, hasta un 20 - 25\% de las pacientes puede desarrollar obstrucción del TSVI y/o insuficiencia mitral aguda, pudiendo llevar a una falla cardiaca aguda, edema pulmonar y shock cardiogénico. Los factores que más se relacionan a la asociación de la cardiomiopatía de Tako tsubo y obstrucción del TSVI se enumeran en la Tabla 1. Frente a este cuadro, el tratamiento debe considerar vigilancia estricta en cuidados intensivos, inicio de fármacos betabloqueadores, volemización y evitar fármacos inotrópicos[5].

En relación al período perioperatorio, se ha descrito una incidencia de la cardiomiopatía de Takotsubo de 1/6.700 intervenciones quirúrgicas. Aproximadamente, $10 \%$ de los casos de Tako tsubo en USA, se relacionan con cirugía no cardiaca y en Japón, se ha descrito que $16,7 \%$ de los casos intrahospitalarios recibieron anestesia general en los últimos 7 días. En una revisión narrativa sobre casos de cardiomiopatía de Takotsubo perioperatorio, lograron identificarse 131 casos publicados[1]. En cuanto al tipo de intervención quirúrgica identificada se encontró gran variedad: cirugía gastrointestinal, cirugía cardiotoráxica, cesárea, cirugía ortopédica, trasplante renal y hepático. La técnica anestésica utilizada en $60 \%$ de los casos fue anestesia general. En relación al momento de aparición, se identificó que 5\% ocurrieron previo a la inducción, 37\% durante la mantención anestésica y

Tabla 1. Factores de riesgo para la asociación entre cardiomiopatía de Takotsubo y obstrucción del TSVI[5],[7]
Edad avanzada
Historia de hipertensión arterial
Hipertrofia septal
Insuficiencia mitral
SAM de la válvula mitral
Inestabilidad hemodinámica


$58 \%$ postoperatorio. Durante este período es más habitual que se presente con síntomas de falla cardiaca, shock cardiogénico, arritmias y paro cardiorrespiratorio[1].

Si bien, no ha podido aislarse una causa directa entre el procedimiento quirúrgico, la patología de base o la anestesia en sí, será el anestesiólogo el encargado del manejo inicial de estos pacientes y de sus potenciales complicaciones asociadas. Frente a un episodio de hipotensión no controlada, una vez descartadas las causas clásicas, es importante que el anestesiólogo se plantee causas poco frecuentes, dentro de las cuales la cardiomiopatía de Tako tsubo deberá encontrarse dentro de los diagnósticos diferenciales, especialmente ante pacientes con predisponentes como se ejemplifica en el caso clínico presentado: mujer, postmenopáusica, en la sexta década de la vida, factor estresante identificable y con factores de riesgos para presentar una complicación como fue la obstrucción TSVI por SAM (estos son: HVI, hipovolemia, estimulación adrenérgica, isquemia miocárdica por Tako tsubo).

\section{Ideas para recordar}

- La hipotensión perioperatoria es una complicación muy común y de causas conocidas. Sin embargo, cuando éstas han sido descartadas, es necesario comenzar a plantearse causas menos frecuentes. Dentro de los diagnósticos cardiológicos, la cardiomiopatía de Takotsubo es más común de lo esperado. Suele ser una condición reversible y benigna, sin embargo, no está libre de complicaciones que pudiesen derivar en falla cardiaca y shock cardiogénico, como la obstrucción del TSVI por SAM.

- El anestesiólogo se verá enfrentado a este diagnóstico en contexto perioperatorio, en procedimientos fuera de pabellón y en unidades de paciente crítico. Por lo tanto, debiese ser considerado como diagnóstico diferencial en pacientes que presenta inestabilidad hemodinámica inesperada y de difícil manejo.

- Es importante destacar el uso de la ecocardiografía perioperatoria como herramienta diagnóstica frente a casos de hipotensión sostenida que no responde a manejo habitual ya que permitirá guiar la terapia de forma más específica y evitar tratamientos que pudiesen ser perjudiciales para el cuadro clínico enfrentado.

\section{Referencias}

1. Hessel EA. Takotsubo cardiomyopathy and its relevance to anesthesiology: a narrative review. J Can Anesth. 2016;63(9):105974. https://doi.org/10.1007/ s12630-016-0680-4.

2. Pelliccia F, Kaski JC, Crea F, Camici PG. Pathophysiology of Takotsubo Syndrome. Circulation. 2017 Jun;135(24):242641. https://doi.org/10.1161/ CIRCULATIONAHA.116.027121 PMID:28606950

3. Agarwal S, Sanghvi C, Odo N, Castresana MR. Perioperative takotsubo cardiomyopathy: implications for anesthesiologist. Ann Card Anaesth. 2019 JulSep;22(3):309-15. https://doi. org/10.4103/aca.ACA_71_18

\section{PMID:31274495}

4. Concha M, Mertz KV. "Systolic anterior motion" (SAM): una causa infrecuente de hipotensión severa en el perioperatorio de cirugía no cardiaca. Revista Médica de Chile. SciELO Agencia Nacional de Investigacion y Desarrollo (ANID); 2012 Aug;140(8):10469. http://dx.doi.org/10.4067/ s0034-98872012000800012.

5. De Backer O, Debonnaire P, Gevaert S, Missault L, Gheeraert $P$, Muyldermans L. Prevalence, associated factors and management implications of left ventricular outflow tract obstruction in takotsubo cardiomyopathy: a two-year, two-center experience. BMC Cardiovasc Disord. 2014 Oct;14(1):147. https://doi. org/10.1186/1471-2261-14-147
PMID:25339604

6. Lasala JD, Tsai J, RodriguezRestrepo A, Ata SM, Sepesi B. Systolic anterior motion of the mitral valve-the mechanism of postural hypotension following left intrapericardial pneumonectomy. Journal of Thoracic Disease. AME Publishing Company; 2017 Apr;9(4):E354-E357. http://dx.doi.org/10.21037/ jtd.2017.03.117

7. Wu Y, Fan W, Chachula L, Costacurta G, Rohatgi R, Elmi $F$. Left ventricular outflow track obstruction and mitral valve regurgitation in a patient with takotsubo cardiomyopathy. J Community Hosp Intern Med Perspect. 2015 Dec;5(6):29419. https://doi.org/10.3402/jchimp. v5.29419 PMID:26653691 